\title{
Der lange Weg zum Psychotherapeutengesetz
}

\author{
Vier Stationen in drei Jahrzehnten
}

\author{
Dietmar Schulte
}

Ruhr-Universität Bochum

\begin{abstract}
Zusammenfassung: In diesem Beitrag soll die wechselhafte Geschichte des Gesetzes anhand historischer Quellen sowie eigener Unterlagen und Erinnerungen nachvollzogen werden. Dabei ergeben sich fünf historische Phasen. Zeit des Aufbruchs: In den 1960er Jahren traten durch die Entwicklung der Verhaltenstherapie als einem psychologischen Therapieansatz wesentliche Veränderungen des Selbstverständnisses und der Tätigkeitsbereiche der Klinischen Psychologen ein. Der BDP beschließt, eine gesetzliche Regelung für eine selbstständige Tätigkeit eines „Fachpsychologen für Klinische Psychologie“ anzustreben. 1973 empfiehlt die Psychiatrie Enquete-Kommission ein Gesetz für nichtärztliche Psychotherapeuten als eine der Sofortmaßnahmen. Erster Anlauf (1974-1978): Die Bundesregierung beginnt 1974 mit der Arbeit. Die Grundzüge werden allgemein begrüßt. Als erst vier Jahre später der Referentenentwurf vorgestellt wird, wird dieser jedoch mehrheitlich abgelehnt. „Interregnum“ (1978-1989): Das BMG stellt die Arbeit am Gesetz ein. Das Bundessozialministerium unternimmt seinerseits Maßnahmen zur Verbesserung der psychotherapeutischen Versorgung. Die Verhaltenstherapie wird Kassenleistung und die KBV fördert und akkreditiert private Ausbildungsinstitute für Verhaltenstherapie. Parallel dazu fördert die Föderation Deutscher Psychologenverbände universitäre Weiterbildungsgänge und akkreditiert diese. Ein von Gesundheitsministerin Ursula Lehr beauftragtes Gutachten empfiehlt 1991 ein Gesetz. Dies zieht Abwehrreaktionen der Ärzteschaft nach sich. Zweiter Anlauf (1993-1995): Das BMG beginnt erneut mit der Arbeit, intensiv begleitet von den inzwischen vielfältigen Berufs-und Fachverbänden. Das Gesetz scheitert im Bundesrat an der sogenannten Zuzahlungsregelung. Dritter Anlauf (1996-1999): 1997 beginnt das BMG erneut mit der Arbeit, beschränkt auf das Sozialrecht. Die Zuzahlungsregelung wird in einem gesonderten Gesetz geregelt. Am 06.03.98 stimmt als letzte Instanz der Bundesrat zu; der Einspruch gegen das Zuzahlungsgesetz wird mit einer Stimme Mehrheit abgelehnt.
\end{abstract}

Schlüsselwörter: Psychotherapeutengesetz, Geschichte des Psychotherapiegesetzes, Entwicklung der Klinischen Psychologie, Professionalisierung

\section{The Long Way to the Psychotherapist Law}

Abstract: In this article, the changing history of the psychotherapist law in Germany will be reconstructed based on historical sources as well as the author*s personal documents and recollections Five historical phases can be differentiated. Time of awakening: In the 1960s, the development of behavior therapy as a psychological therapeutic approach brought about significant changes in the self-image and fields of activity of clinical psychologists. The Berufsverband deutscher Psychologen (BDP). decided to strive for legal regulation of the independent position of specialist psychologist for clinical psychology. In 1973 the Psychiatry Enquete Commission recommended a law for nonmedical psychotherapists as one for immediate action. First attempt (1974-1978): The federal government began work in 1974. The main features of the new law were generally welcomed. However, when the draft bill was not presented until 4 years later, it was rejected by a majority. Interregnum (1978-1989): The BMG stopped work on the law. For its part, the Federal Ministry of Social Affairs (KBV) took steps to improve psychotherapeutic care. Behavior therapy was covered by health insurance, and the KBV promoted and accredited private training institutes for behavior therapy. In parallel, the Federation of German Psychologists' Associations promoted and accredited university continuing education courses. A report commissioned by Health Minister Ursula Lehr recommended a law in 1991. This led to defensive reactions by the medical profession. Second attempt (1993-1995): The BMG started work again, intensively supported by the now diverse professional and therapy associations. The law failed in the Bundesrat because of the so-called co-payment regulation. Third attempt (1996-1999): In 1997, the BMG started work again, limited to social law. The co-payment regulation was regulated in a separate law. On March 6, 1998, the Bundesrat, in the final instance, voted in favor; the appeal against the co-payment law was rejected by a majority of one vote.

Keywords: psychotherapist law, history of psychotherapy law, development of clinical psychology, professionalization 
Dreißig Jahre bis zum Psychotherapeutengesetz - das ist ein langer Zeitraum. Zwangsläufig sind meine Erinnerungen durch meine persönlichen Erfahrungen während dieser Zeit geprägt. Ich werde dies im Folgenden nicht verschweigen, sondern gelegentlich sogar ausdrücklich darauf hinweisen. Die Bemühungen um ein Psychotherapeutengesetz haben mich seit 1970 beschäftigt. Zu diesem Zeitpunkt, zwei Jahre nach meinem Diplom in Münster, wurde ich Mitglied des Vorstands der Sektion Klinische Psychologie des Berufsverbandes Deutscher Psychologen (BDP), damals der einzige Berufsverband. Danach war ich bis zur Verabschiedung des Gesetzes und danach in verschiedenen anderen Funktionen tätig, vor allem für die Deutsche Gesellschaft für Psychologie (DGPs). In diesen 30 Jahren hat sich eine Vielzahl von Personen für das Psychotherapeutengesetz engagiert. Da ich unmöglich allen gerecht werden kann, verzichte ich weitgehend darauf, im Folgenden Namen zu nennen.

\section{Wie alles begann}

In den 1960er Jahren gab es das Prüfungsfach Klinische Psychologie noch nicht, es hieß z.B. in Münster „Psychagogik und Tiefenpsychologie“. Das typische Tätigkeitsfeld der „Kliniker“ waren Erziehungsberatungsstellen. Es ging um Diagnostik und anschließende Beratung. Psychotherapie war weder möglich noch erlaubt, lediglich tiefenpsychologisch geprägte Kinder-(Spiel-)Therapien. Aber es zeichnete sich eine wichtige und entscheidende neue Entwicklung ab.

Zum einen wurde die Klientenzentrierte Gesprächspsychotherapie, entwickelt von dem Theologen und Psychologen Carl Rogers (1951), von Reinhard Tausch (1968) in Hamburg bekannt gemacht. Zum anderen kam es zur Entwicklung einer neuen Psychotherapie, der Verhaltenstherapie, anders als alle andere Verfahren nicht aus der Praxis heraus entwickelt, sondern aus der Wissenschaft. In den 1950er und vor allen dann in den 1960er Jahren erschienen erst einzeln, dann immer mehr Arbeiten. Dieser neue Ansatz wurde in Deutschland vor allem durch die Psychologischen Institute der Universitäten München und Münster sowie dem Max-Plank Institut für Psychiatrie in München aufgegriffen (Fiedler, 2010; vgl. den Beitrag von Malich in diesem Heft). Im Rahmen des Diplomstudiengangs Psychologie wurden erste verhaltenstherapeutische Lehrveranstaltungen angeboten, die wir in
Münster, ab 1974 dann in Bochum, zu einem dreisemestrigen Curriculum ausbauten. Die Nachfrage war groß, nicht nur seitens der Studierenden. Viele Berufstätige fragten nach, ob sie nicht an den Lehrveranstaltungen teilnehmen könnten oder ob wir ihnen nicht wenigstens einen Studierenden als Praktikanten schicken könnten, damit sie von diesem diese neue Verhaltenstherapie lernen könnten.

In wenigen Jahren verbreiteten sich diese neuen Therapieverfahren in der Praxis (Schulte, 1971). 1968 wurden die Gesellschaft zur Förderung der Verhaltenstherapie (GVT) und die Gesellschaft für wissenschaftliche Gesprächspsychotherapie (GWG) gegründet. In den siebziger Jahren kamen dann Verbände für andere, humanistische und systemische Therapierichtungen, hinzu.

Die Auswirkungen dieser Entwicklung waren gravierend:

- Die Praxis veränderte sich. An die Stelle von Diagnostik trat Therapie.

- Das Selbstverständnis der Psychologen und Psychologinnen veränderte sich. Therapeutische Tätigkeit war jetzt psychologische Tätigkeit. Psychologen und Psychologinnen erlebten sich als kompetent, aber sie durften nicht oder nur unselbstständig tätig werden.

- Und das Tätigkeitsfeld veränderte sich zunehmend zur Tätigkeit in eigener Praxis.

Ein paralleler Entwicklungsstrang hatte 1964 begonnen (Schildt, 2007), als der Bundesgerichtshof feststellte, dass psychische Störungen als behandlungsbedürftige Krankheiten zu betrachten seien. 1967 wurde dann von der Kassenärztlichen Bundesvereinigung (KBV) die Psychoanalyse als Kassenleistung anerkannt, abrechenbar von ärztlichen Psychoanalytikern. Da die Zahl ärztlicher Psychotherapeuten und Psychotherapeutinnen bei weitem nicht den Bedarf decken konnte, wurde 1972 das Delegationsverfahren (Farber \& Haarstrick, 1989) für entsprechend weitergebildete Psychologinnen und Psychologen beschlossen: der Arzt delegierte die Durchführung der Therapie an einen Psychologen oder eine Psychologin. Aber er stellte nicht nur selber die Diagnose und Indikation, sondern in den ersten Jahren rechnete er auch mit der Kasse ab und gab dann Geld an den Behandler weiter. Psychologen zählten damit zu den Heilhilfsberufen. In diesem Zusammenhang wurde der nur gelegentlich verwendete Begriff Tiefenpsychologische Verfahren offiziell eingeführt - aus berufspolitischen Gründen ${ }^{1}$ : Tiefenpsychologische Verfahren durften von den

\footnotetext{
Aus diesem Grund hat der Wissenschaftliche Beirat Psychotherapie die auch international ungebräuchliche Unterscheidung in Psychoanalyse und Tiefenpsychologische Verfahren verworfen und 2004 nur die Wissenschaftliche Anerkennung für die Psychodynamische Psychotherapie überprüft (https://www.wbpsychotherapie.de).
} 
Psychologinnen und Psychologen durchgeführt werden, die Psychoanalyse blieb den Ärztinnen und Ärzten vorbehalten.

Weder die Ärzte noch die Psychologen boten in ausreichendem Maße psychotherapeutische Behandlungen für Kinder und Jugendliche an, so dass etwas später dann auch Pädagogen und Pädagoginnen und andere zum Delegationsverfahren zugelassen wurden, beschränkt auf die analytische Kinder- und Jugendlichen Psychotherapie.

Auch dies reichte nicht, um den Bedarf zu decken. Die KBV wünschte die neue Verhaltenstherapie als Kassenleistung. 1971 begannen entsprechende Gespräche der KBV mit dem Deutschen Berufsverband der Verhaltenstherapeuten (DBV).

1971 wurde der DBV als berufspolitische Ergänzung der GVT gegründet, damit diese ihre Anerkennung als gemeinnützig nicht verlor. Mitglied konnten nur ausgebildete Verhaltenstherapeuten werden. Beeinflusst durch die antiautoritäre Entwicklung der 1968er Jahre kam es im Vorstand der GVT zu heftigen Auseinandersetzungen mit Studierenden, die zum Rücktritt von Johannes Brengelmann als Vorsitzendem führten (Daiminger, 2007). Auf der Mitgliederversammlung anlässlich des Verhaltenstherapiekongresses in Münster 1972 wurde in Abwesenheit Rudolf Cohen zum neuen Vorsitzenden gewählt, der dann die Wahl jedoch nicht annahm. Diese Krisensituation nutze eine Gruppe Münchener Studenten mit Verbindung zu dem Sozialpsychologen Heiner Keupp, sich vom Gericht als Notvorstand einsetzen zu lassen. Die Satzung wurde verändert, so dass auch Studierende Mitglied der GVT werden konnten. Der DBV wurde massiv als berufsständisch kritisiert, und der Zugang zu den bislang gemeinsamen Mitteilungen und zur Adressendatei der Mitglieder wurde ihm verweigert. Auf dem Kongress 1973 in München wurde dann mit Peter Gottwald an der Spitze ein neuer Vorstand der GVT gewählt. Nach zwei Jahren begannen Gespräche über eine Wiederannäherung der beiden Vereine, die 1976 mit einem Zusammenschluss und der Gründung der Deutsche Gesellschaft für Verhaltenstherapie, DGVT, endeten. In der Satzung wurden sowohl die gesundheitspolitischen Zielsetzungen als auch die Förderung der Verhaltenstherapie und die berufspolitischen Belange verankert. Auf der ersten Mitgliederversammlung nach dem Zusammenschluss wurden diese letztgenannten Ziele jedoch wieder aus der Satzung gestrichen. Die damit entstandene Lücke in Hinblick auf die Förderung der Verhaltenstherapie und ihre Forschung wurde 1985 durch die Gründung der Deutsche Gesellschaft für Verhaltensmedizin und Verhaltensmodifikation (DGVM) geschlossen.

Aber die Durchführung der Verhaltenstherapie sollte ebenfalls nur im Delegationsverfahren möglich sein. Die Verhaltenstherapeuten lehnten das ab. Sie wünschten ei- ne selbständige und eigenverantwortliche Tätigkeit. Nach einigen Jahren wurden die Verhandlungen daher abgebrochen. Allerdings ließen sich viele Psychologinnen und Psychologen trotzdem in eigener Praxis nieder. Wesentlicher Grund dafür war - nach einem drastischen Anstieg der Studierendenzahlen seit Mitte der 1960er Jahre - eine zunehmende (Übergangs-)Arbeitslosigkeit (Hörmann \& Nestmann, 1985). Die Niederlassung in eigener Praxis war oft die einzige Möglichkeit der Berufstätigkeit. Die Kassen begannen zunehmend, Verhaltenstherapien von Psychologen und Psychologinnen außerhalb des Delegationsverfahrens - sofern kein anerkannter „RichtlinienPsychotherapeut" vermittelt werden konnte - nach dem sogenannten Erstattungsverfahren zu bezahlen. Dabei mussten die Patienten in Vorleistung treten und die Kasse erstattete ihnen später die Kosten (Abbildung 1).

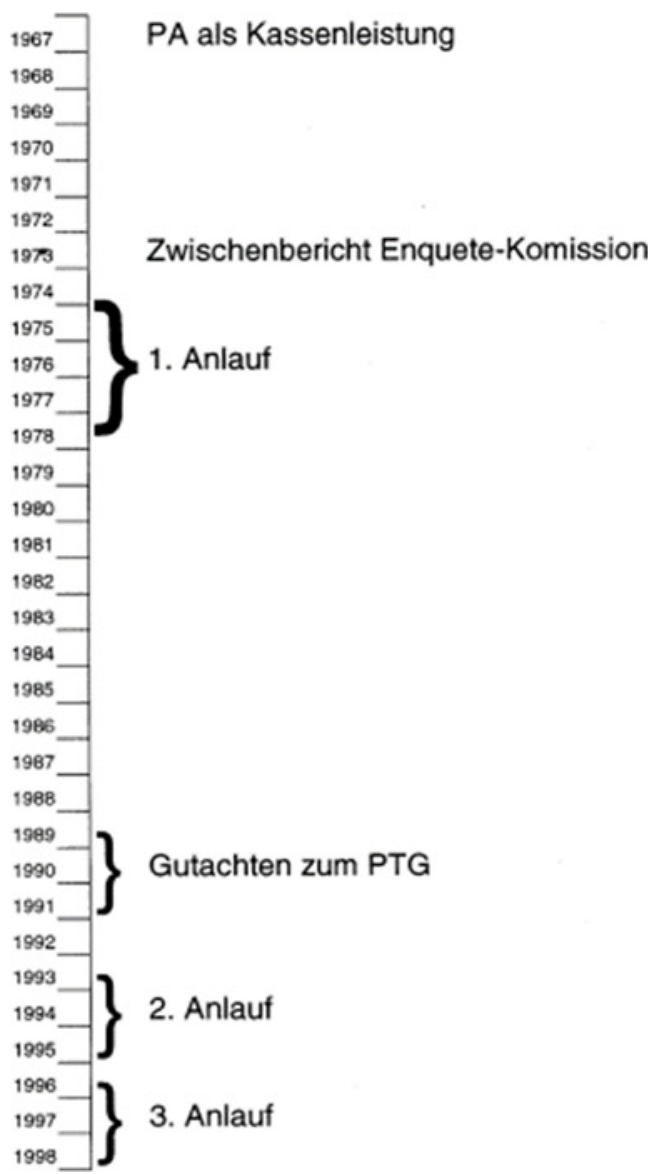

Abbildung 1. Ablauf der Vorgeschichte des Psychotherapeutengesetzes. 


\section{Erster Anlauf zum Psychotherapeutengesetz (1968-1978)}

Die Psychologinnen und Psychologen strebten eine selbstständige und eigenverantwortliche Tätigkeit als Klinische Psychologen bzw. Psychologinnen an. Das war im Berufsverband Deutscher Psychologen (BDP) allerdings nicht unumstritten, da - bereits damals - eine Zweiteilung der Psychologenschaft befürchtet wurde. Doch 1968 beschloss die Mitgliederversammlung des BDP mehrheitlich in Tübingen, eine "gesetzliche Sonderregelung für Klinische Psychologen" anzustreben. 1970 traf die Mitgliederversammlung der Sektion Klinische Psychologie einen entsprechenden Grundsatzbeschluss und entschied 1971, einen „Fachpsychologen für Klinische Psychologie“ einzurichten.

Der erste Schritt war, ein Berufsbild und einen Ausbildungsplan für einen solchen Fachpsychologen zu entwickeln. Dazu bildete die Sektion Klinische Psychologie eine Arbeitsgemeinschaft mit den damals existierenden Therapieverbänden: Deutsche Gesellschaft für Psychotherapie und Tiefenpsychologie (DGPT), Deutsche Gesellschaft für Individualpsychologie (DGIP), der GwG und dem DBV. 1973 wurden Berufsbild und Ausbildungsplan veröffentlicht (erstmals mit dem neuen Logo des BDP, aber leider seitenverkehrt, s. Abbildung 2).

Ein wesentlicher Anstoß in Richtung einer solchen gesetzlichen Regelung erfolgte im Rahmen von zwei Bundestags-Anhörungen, 1970 und 1971, zur Lage der Psychiatrie in Deutschland, angeregt unter anderem durch eine Psychiatriereform in den USA durch Kennedy (Söhner, Fangerau \& Becker, 2017). Bei diesen Anhörungen wurden auch zwei Psychologen, Johannes Brengelmann, MPI für Psychiatrie in München, und Erna Duhm, Psychologisches Institut der Universität Göttingen, gehört. Sie berichteten beide über die neue Entwicklung der Verhaltenstherapie und forderten, Klinische Psychologen zur Ausübung der Heilkunde zuzulassen. 1971 wurde vom Bundestag eine Psychiatrie-Enquete-Kommission eingesetzt. Psychologen oder Psychologinnen waren zwar für die Arbeitskreise, nicht aber für die Kommission vorgesehen. Der genannten Arbeitsgemeinschaft gelang es schließlich, dass zumindest ein Psychologe, Rudolf Cohen, Professor an der Universität Konstanz, Mitglied wurde.

1973 veröffentlichte die Enquete-Kommission einen Zwischenbericht, in dem unter anderem als Sofortmaßnahme ein Gesetz für „nichtärztliche Psychotherapeuten“ empfohlen wurde (Deutscher Bundestag, 1973).

Der BDP machte das Berufsbild und den Ausbildungsplan für einen Fachpsychologen bekannt. Die Bundesärztekammer richtete daraufhin unter Leitung von Dr.
Paul Erwin Odenbach eine Kommission mit dem Namen „Randgebiete der Medizin“ ein - die Randgebiete waren die Psychologie. Der DBV wurde zu einer Sitzung eingeladen. Meine persönliche Erinnerung an diese Sitzung war: Obwohl Vorsitzender des DBV durfte ich als Psychologe nicht in den Sitzungsraum, sondern musste vor der Tür warten. Ein Vorstandskollege, der Arzt war, ging in die Sitzung und kam gelegentlich zu mir heraus, um über den Fortgang der Besprechung zu berichten.

Das Bundesministerium für Jugend, Familie und Gesundheit (BMJFG) übernahm 1974 die Forderung der Enquete-Kommission und führte eine Sachverständigenanhörung durch, an der ich als einziger Psychologe teilnehmen konnte. Bei dieser Anhörung wurden Grundzüge für ein Psychotherapeutengesetz formuliert, die im Wesentlichen mit den Vorstellungen der Psychologen übereinstimmten (Auszüge der Niederschrift über die Sachverständigenanhörung vom 28. Februar 1974 im BMJFG werden von Wittchen \& Fichter, 1980, zitiert). BDP und Psychotherapieverbände stimmten diesen Grundzügen zu, und auch der Deutsche Ärztetag fasste im gleichen Jahr einen entsprechenden Beschluss. Daraufhin begann das Ministerium mit der Arbeit an einem Referentenentwurf, unterstützt von uns durch manche Besprechungen im Ministerium.

Nunmehr konnte das Gesetz kommen - aber es dauerte (Schulte, 1977). Ein wesentlicher Grund dafür war folgender: 1975 legte die Enquetekommission ihren Schlussbericht vor (Deutscher Bundestag, 1975), in dem auch die Situation der Psychotherapie dargestellt wurde, allerdings beschränkt auf die Tätigkeit ärztlicher und tiefenpsychologisch weitergebildeter nicht-ärztlicher Psychotherapeuten und Psychotherapeutinnen. Daher beauftragte das Bundesministerium für Jugend, Familie und Gesundheit (BMJFG) im Herbst 1976 den Direktor des MaxPlank-Instituts für Psychiatrie, Detlev Ploog, „offene Fragen im Zusammenhang mit Vorarbeiten zum Gesetzentwurf über den Beruf des ,nichtärztlichen Psychotherapeuten“" (Wittchen \& Fichter, 1980, S.14) zu klären. Im Wesentlichen sollten Tätigkeitsbereiche und Qualifikation ,nichtärztlichen Psychotherapeuten' erfasst werden. Die Ergebnisse der umfangreichen Erhebungen aus den Jahre 1977 und 1978 wurden 1980 veröffentlicht (Wittchen \& Fichter, 1980).

Insgesamt vier Jahre dauerte es, bis dann endlich von Gesundheitsministerin Antje Huber, SPD, der Referentenentwurf vorgelegt wurde. Er folgte im Wesentlichen der Sachverständigenanhörung von 1974. Die Anerkennung sollte auf vier Therapierichtungen beschränkt sein: Psychoanalyse/Tiefenpsychologie, Verhaltenstherapie, Gesprächspsychotherapie und Individualpsychologie (nach Alfred Adler). Das BMJFG führte im September 1978 zwei Anhörungen durch. Das Ergebnis war niederschmetternd, 


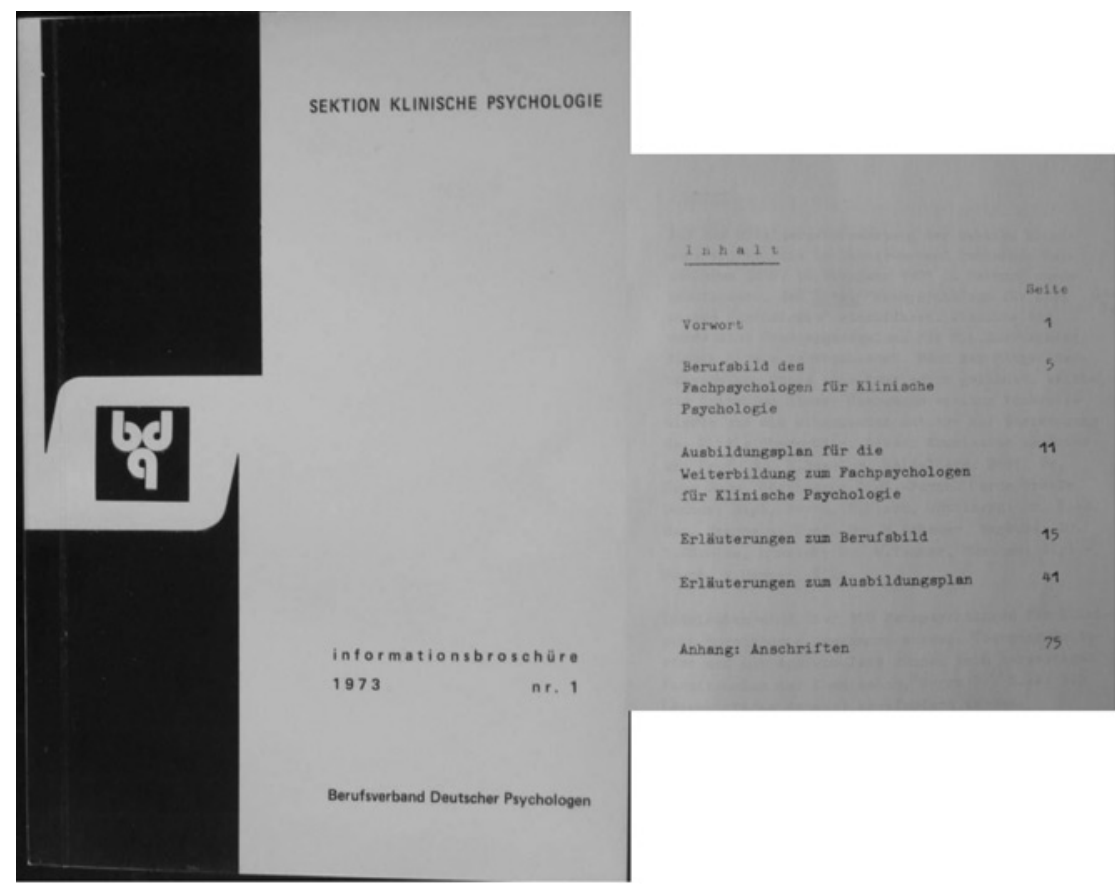

Abbildung 2. Veröffentlichung von Berufsbild und Weiterbildungsplan des Fachpsychologen für Klinische Psychologie, 1973. der Entwurf wurde weitgehend abgelehnt. Nur die Psychoanalytiker, die Ärzte und die Hochschullehrer für Klinische Psychologie stimmten zu. Was war in diesen vier Jahren passiert, so dass es nun zu dieser Ablehnung kam?

Abgelehnt wurde der Entwurf von den sogenannten Plattformverbänden, der Deutschen Gesellschaft für Verhaltenstherapie (DGVT), der GWG und der Deutschen Gesellschaft für soziale Psychiatrie (DGSP). 1976 hatte die Enquete-Kommission ihren Schlussbericht mit der Empfehlung weitgehender Reformen veröffentlicht, die die gesamte Versorgung psychisch Kranker, vor allem die stationäre Unterbringung und Behandlung, umfasste. Die Plattformverbände vertraten im Sinne des Schlussberichtes die Auffassung, dass nunmehr eine grundlegende Psychiatriereform an der Zeit sei und nicht ein separates Berufsgesetz für Psychologinnen und Psychologen.

Abgelehnt wurde der Entwurf auch - ausgerechnet von der Sektion Klinische Psychologie des BDP. Warum? 1976 forderte die KBV von den Kassen, keine Bezahlung mehr außerhalb des Delegationsverfahrens zuzulassen (Schild, 2007). Die Existenz vieler niedergelassener Psychologen, die über das Erstattungsverfahren abrechneten, war bedroht. Sie übernahmen die Führung im BDP und in der Sektion Klinische Psychologie. Da die meisten keine gesonderte Psychotherapieausbildung oder Anerkennung als Fachpsychologe nachweisen konnten, forderten sie die Heilkundezulassung für alle Psychologen und lehnten daher den Referentenentwurf ab.

Abgelehnt wurde der Entwurf auch vom Bundessozialministerium, da die Finanzierung nicht geklärt war. Der
Referentenentwurf enthielt keine sozialrechtlichen Regelungen. Grund dafür war, dass die finanziellen Regelungen des Gesundheitssystems nach der Reichsversicherungsordnung (heute Sozialgesetzbuch V) nicht dem Gesundheitsministerium, sondern dem Bundessozialministerium oblagen. Erst 1992, mit Gesundheitsminister Horst Seehofer, der zuvor Parlamentarischer Staatssekretär im Sozialministerium war, wurde die entsprechende Abteilung des Sozialministeriums für das SGB V in das Gesundheitsministerium überführt.

\section{Jahre „Interregnum“ (1978-1989)}

Die Konsequenzen des Scheiterns waren gravierend und nachhaltig. Die Enttäuschung und Verärgerung der zuständigen Mitarbeiterinnen und Mitarbeiter des BMJFG war verständlicherweise groß. Das BMJFG stellte die Arbeit am Gesetz „endgültig“ ein (für 11 Jahre, bis 1989). Die damals zuständige Ministerialrätin Marilene Schleicher sagte mir, dass es, solange sie im Ministerium sein werde, kein Psychotherapeutengesetz geben werde. Sie sollte Recht behalten; kurz vor Verabschiedung des Gesetzes verließ sie vorzeitig das Ministerium.

Besonders gravierend waren die sozialrechtlichen Konsequenzen. Das Sozialministerium hatte den Entwurf abgelehnt, sah sich nun aber in der Pflicht, angesichts des tatsächlich bestehenden Bedarfs an Psychotherapie von sich aus tätig zu werden. Die KBV nahm die Verhandlun- 
gen zur Zulassung der Verhaltenstherapie als Kassenleistung wieder auf, aber die Verhaltenstherapeuten bestanden weiterhin darauf, nicht im Rahmen des Delegationsverfahrens tätig zu werden. Bedeutete das doch eine unselbständig Arbeit in Abhängigkeit eines Arztes oder einer Ärztin, die sie im Extremfall selber ausgebildet hatten. Schließlich sagte mir der federführende ärztliche, psychoanalytische Kollege, Rudolf Haarstrick, dass sie jetzt die Verhaltenstherapie ohne uns (die Verhaltenstherapeuten) zunächst für zwei Jahre zulassen würden. Wir würden sehen: nach zwei Jahren würden sie so viele Psychologen im Delegationsverfahren haben, dass sie dann die Zulassung endgültig beschließen können. Er sollte Recht behalten. Die KBV beschloss 1980 den entsprechenden Vertrag mit den Ersatzkassen. Noch im gleichen Jahr wurde der Fachverband für Klinische Verhaltenstherapie (FKV) gegründet. 1984 gründete sich die Vereinigung der Kassenpsychotherapeuten, die dann zum Gesprächspartner der KBV wurde (Mehring, 1992). 1987 wurde Verhaltenstherapie dann auch Leistung der Primärkassen.

Die Zulassung von Verhaltenstherapeuten und -therapeutinnen zum Delegationsverfahren erforderte den Nachweis einer qualifizierten Ausbildung, aber die Weiterbildung in Verhaltenstherapie lag zu dem Zeitpunkt im Argen. Der deutsche Berufsverband der Verhaltenstherapeuten (DBV) hatte zwar bereits sehr früh curricular strukturierte Weiterbildungen angeboten, anfangs vom Landesarbeitsamt NRW gefördert (Fiedler, 2010). Aber die durch Zusammenschluss mit der GVT entstandene neue DGVT lehnte diese Weiterbildung ab und förderte eine Weiterbildung in Form eines Selbststudiums in Arbeitskreisen, ohne Einbezug erfahrenerer Therapeutinnen oder Therapeuten (Daiminger, 2007). Diese Form von Weiterbildung fand allerdings außerhalb des Verbandes keine Akzeptanz. Daher begann die KBV als zweite sozialrechtliche Maßnahme damit, private Ausbildungsinstitute für Verhaltenstherapie nach dem Modell der psychoanalytischen Ausbildung zu fördern und anzuerkennen. Die Institute mussten von einem Arzt oder einer Ärztin und einem Psychologen oder einer Psychologin geleitet werden.

Die KBV richtete zu diesem Zweck eine Anerkennungskommission ein. Da jedoch rechtlich gesehen die Ausbildung nicht zum Aufgabenbereich der KBV gehörte, konnte die Kommission keine Ablehnungen von Anträgen auf Anerkennung aussprechen. Es wurden lediglich Hinweise gegeben, an welchen Punkten die geplante Ausbildung nicht den Anforderungen entsprechen würde. Die Anträge wurden dann so oft korrigiert, bis schließlich von der Kommission eine Anerkennung ausgesprochen wurde. Rechtlich bedeutete das, dass die Absolventen dieser Ausbildung zur Verhaltenstherapie im Delegationsverfahren zugelassen wurden. Die von der KBV aner- kannten Institute schlossen sich im Fachverband für Klinische Verhaltenstherapie (FKV) und in der Deutschen Akademie für Verhaltenstherapie (DAVT) zusammen. Beide fusionierten 1992 zum Deutschen Fachverband für Verhaltenstherapie (DVT).

Parallel dazu gab es eine zweite Entwicklung zur Verbesserung der Ausbildung von Klinischen Psychologinnen und Psychologen. Die klinische Ausbildung während des Psychologiestudiums war an fast allen Universitäten gekürzt worden, nachdem die DGPs 1983 ihre Rahmenprüfungsordnung verändert und für den zweiten Studienabschnitt (wieder) Ausbildungen in allen drei Anwendungsfächern der Psychologie vorsah. Eine Schwerpunktbildung in Klinischer Psychologie, die die Rahmenprüfungsordnung von 1972 zugelassen hatte, war damit nicht mehr möglich (Krampen, 1992).

Als Ausgleich wollte der Vorstand der DGPs weiterbildende Studiengänge in den Anwendungsfächern fördern (Schulte, 1984). Dementsprechend planten wir in Bochum einen zweijährigen weiterbildenden Studiengang zum Klinischen Psychologen, der 1982 von der DGPs empfohlen wurde (Schulte, 1987). Dies sollte im Rahmen eines offiziellen Bund-Länder Modellversuchs geschehen. Über drei Jahre lang bemühten wir uns um die Einrichtung dieses Modellversuchs. $\mathrm{Zu}$ beteiligen waren vier Ministerien: das Wissenschaftsministerium und das Gesundheitsministerium des Landes NRW und die entsprechenden Ministerien des Bundes. Am Ende scheiterte der Antrag am Votum der erwähnten Mitarbeiterin aus dem BMJFG, da die Psychologen ja keine Heilkunde ausüben dürften. Ich stellte den Antrag noch einmal, und es dauerte noch einmal vier Jahre. Am 21.10.1988 wurde er bewilligt (Schulte, 1993a), auf Drängen des BMJFG mit dem Hinweis, dass damit kein Präjudiz für ein Psychotherapeutengesetz geschaffen würde. Egal - das Psychotherapeutengesetz war wieder im Gespräch. Die oben genannte Ministerialrätin des BMJFG war dann Mitglied des Beirats, der den Modellversuch begleitete. Der erste Jahrgang begann im Oktober 1989. Nach erfolgreichem Abschluss erhielten die Teilnehmer ein Zertifikat.

Universitäre Weiterbildungsgänge wurden dann nachund nach auch an anderen Universitäten eingerichtet, und die Föderation Deutscher Psychologenverbände (DGPs und BDP) übernahm das Konzept des Modellversuchs, beschloss 1989 Qualitätskriterien für solche universitären Weiterbildungsgänge und richtete eine Anerkennungskommission ein. Allerdings wurde den Absolventen und Absolventinnen dieser universitären Weiterbildungsgänge der Zugang zum Delegationsverfahren von der KBV verweigert, trotz anfangs anderslautender Zusagen.

Die Tätigkeit der Anerkennungskommission wurde später eingestellt, als sich die Verabschiedung des Gesetzes abzeichnete. Nach Inkrafttreten des Gesetzes wurde 
die Idee wieder aufgegriffen. Die DGPs beschloss, qualifizierte universitäre Ausbildungsgänge, wie sie jetzt hießen, zu akkreditieren. Diese schlossen sich 2003 im Verbund universitärer Ausbildungsgänge für Psychotherapie (unith) zusammen (https://www.klinische-psychologie-psy chotherapie.de/index.php/universitaere-ausbildungsinsti tute).

Eine zweite Strategie, ein Psychotherapeutengesetz zu fördern - neben den Bemühungen um eine nachweislich qualifizierte Ausbildung - betraf das Sozialrecht, konkret das Delegationsverfahren für Verhaltenstherapeuten. Dieser Weg wurde vor allem vom BDP beschritten, später dann mit großem Einsatz vom Deutschen Psychotherapeutenverband (DPTV). ${ }^{2}$ Ziel war es deutlich zu machen, dass die Versorgung von Patienten durch psychotherapeutisch tätige Ärzte und Ärztinnen und Psychologen und Psychologinnen im Delegationsverfahren nicht ausreichend gelingen konnte. Viele Mitglieder des BDP bzw. des DPTV verweigerten sich weiterhin dem Delegationsverfahren und waren nach dem Kostenerstattungsprinzip tätig, nicht zuletzt um den Druck zu erhöhen.

1983 hatte das Bundesverwaltungsgericht bestätigt, dass Psychotherapie Ausübung der Heilkunde sei (Schildt, 2007). Da eine gesetzliche Regelung für Psychologen noch ausstehe, müssten diese eine Zulassung zur Ausübung der Heilkunde nach dem Heilpraktikergesetz von 1939 beantragen. Allerdings sei eine eingeschränkte Überprüfung der erforderlichen Kennnisse möglich, da die Psychologen ja bereits über ein einschlägiges Hochschulstudium verfügten und nur eine eingeschränkte Tätigkeit anstrebten. Niedergelassene Psychologinnen und Psychologen, die es bis dahin abgelehnt hatten, sich als Heilpraktiker anerkennen zu lassen, taten nunmehr diesen Schritt, um rechtliche Konsequenzen zu vermeiden. $\mathrm{Ab}$ gerechnet wurde im Erstattungsverfahren; die Kassen sahen sich aufgrund der weiterhin großen Nachfrage dazu gezwungen. Die Techniker Krankenkasse erklärte sich sogar in einem Vertrag mit dem BDP 1983 bereit, für ihre Patientinnen und Patienten die Abrechnung nach dem Erstattungsverfahren zu erleichtern.

Durch jahrelange Gespräche mit dem Bundesgesundheitsministerium, dem Bundessozialministerium, den Gesundheitspolitikern, den Krankenkassen und den Ärzteverbänden sowie durch entsprechende Pressemitteilungen gelang es in kleinen Schritten, zunehmend die Kritik am Delegationsverfahren zu fördern. Unterstützt vor al- lem von Hans-Joachim Schwarz, Vorsitzender des DPTV, wurde auch die juristische Ebene beschritten, und zwar durch Sozialgerichtsverfahren, angestrebt von Patienten Bremer Psychotherapiepraxen. Das Landessozialgericht Bremen rief schließlich 1980 das Bundesverfassungsgericht an. Dies eröffnete zwar nicht das Verfahren, erklärte aber in einem Beschluss von 1988 das Delegationsverfahren für verfassungskonform, äußerte jedoch Zweifel, ob das Delegationsverfahren den Einbezug von Psychologen in das System der Krankenversicherung befriedigend löse. Es erklärte eine gesetzliche Regelung für heilkundlich tätige Psychologinnen und Psychologen für wünschenswert, wodurch der Gesetzgeber implizit zum Handeln aufgefordert war (Joussen, 2004).

\section{Der zweite Anlauf zum Psycho- therapeutengesetz (1989-1995)}

Am 03. Oktober 1990 fand die deutsche Wiedervereinigung statt, auch für die Gesundheitspolitik eine große Herausforderung. In der DDR hatten sich schon 1954 etwa 30 Klinische Psychologinnen und Psychologen zur „Arbeitsgemeinschaft der Psychologen im Gesundheitswesen der DDR" zusammengeschlossen, ab 1963 wurde ein spezialisiertes Diplom in "Klinischer Psychologie“ angeboten und 1981 wurde der Fachpsychologe für Medizin eingeführt (Rösler, 2011). Die Fachpsychologen durften nun auch offiziell heilkundlich tätig werden. Eine Tätigkeit in privater Praxis war in der DDR nicht möglich, aber mit dem Beitritt der neuen Länder zur Bundesrepublik wuchs trotzdem der Druck, eine Lösung für Psychologinnen und Psychologen zu finden.

Ende 1989 kam Bewegung in die festgefahrene Situation, als Bundeskanzler Helmut Kohl die Psychologin Ursula Lehr als Bundesgesundheitsministerin berief. Sie konnte im Ministerium durchsetzen, dass ein Gutachten zur Frage eines Psychotherapeutengesetzes vergeben wurde. Drei Jahre wurde von der Expertenrunde daran gearbeitet, begleitet von einem Beirat, bis schließlich 1991 das Gutachten vorgelegt wurde (Meyer et al., 1991), das einhellig ein Psychotherapeutengesetz empfahl. Die Hoffnung war groß, dass nun endlich das Gesetz kommen würde. Aber zunächst einmal kam es zu deutlichen Reaktionen

\footnotetext{
Seit Ende der 1980er Jahre gab es im BDP zunehmend Spannungen zwischen dem Präsidium und der Sektion Klinische Psychologie bezüglich der Frage, wer den BDP bei Besprechungen und Außenkontakten im Zusammenhang mit dem Psychotherapeutengesetz vertreten dürfe. 1992 traten die meisten Klinischen Psychologen aus Protest aus dem BDP aus und gründeten den Deutschen Psychotherapeutenverband (DPTV), heute mit der Vereinigung der Kassenpsychotherapeuten zusammengeschlossen zur Deutschen Psychotherapeutenvereinigung (DPtV). Als Reaktion gründete der BDP den VPP (Verband Psychologischer Psychotherapeutinnen und Psychotherapeuten im BDP), dem etwas mehr Autonomie gewährt wurde als den Sektionen.
} 
auf das Gutachten: 1992 beschloss der Deutsche Ärztetag die Umbenennung des Fachgebiets „Psychiatrie“ in „Psychiatrie und Psychotherapie“ und führte zusätzlich das neue Fachgebiet „Psychotherapeutische Medizin“ (ab 2003 „Psychosomatische Medizin und Psychotherapie“) ein. Daraufhin reagierten die Psychologen und Psychologinnen und benannten - aus politischen Gründen - ihre Lehrstühle und die Fachzeitschrift neu in „Klinische Psychologie und Psychotherapie“. Die KBV veröffentlichte eine äußerst kritische und ablehnende Stellungnahme zu dem Gutachten (Anhang „Stellungsnahmen“ zum Forschungsgutachten, Meyer et al., 1991), so das deutlich wurde, dass jetzt der „Kampf“ erst richtig beginnen würde.

Die psychologischen und psychotherapeutischen Berufs-und Fachverbände schlossen sich in der sogenannten „Spiegel AG“ zusammen, um den Gesetzgebungsvorgang zu spiegeln und zu fördern. Die DGPs bildete eine sogenannte Kernkommission, zusammen mit dem BDP, ab 1992 mit der DPTV. Zu den von der Kernkommission entwickelten Strategien zur Förderung der Gesetzgebung gehörten unter anderem eine Emnid-Umfrage der Bevölkerung zur Frage der Zustimmung zu einem Psychotherapeutengesetz (Schulte, 1993b) und eine große Unterschriftenaktion von Psychologie-Professoren.

Der Druck wurde größer. Dazu trug bei, dass das Landessozialgericht NRW die erwähnte Empfehlungsvereinbarung des BDP mit der Techniker Krankenkasse zur Abrechnung im Erstattungsverfahren und eine vergleichbare Vereinbarung, die 1994 die DPTV mit den Betriebs- und Innenkrankenkassen getroffen hatte, auf Antrag der KBV aufhob. Die Begründung war, dass nur die Kassenärztlichen Vereinigungen und die KBV Vertragspartner der Krankenkassen sein könnten. Damit wurde die Kostenerstattung im Rahmen der Psychotherapieversorgung außerhalb der Regelversorgung und der Richtlinientherapien sehr schwierig.

Gegen Ende der Legislaturperiode, 1995, wurde dann endlich vom Bundesministerium für Gesundheit (BMG) unter Horst Seehofer ein Gesetzestext vorgelegt. Doch das Gesetz scheiterte im Bundesrat an der von Seehofer geforderten Zuzahlung: die Kassenpatienten sollten bei einer Psychotherapie einen eigenen Betrag zuzahlen. Dies wurde von den SPD-regierten Ländern aus grundsätzlichen gesundheitspolitischen Gründen abgelehnt.

\section{Der dritte Anlauf zum Psycho- therapeutengesetz (1996-1998)}

Direkt nach der Bundestagswahl legte der Bundesrat mit der Mehrheit der SPD-regierten Länder, gemeinsam mit der Bundestags-SPD-Fraktion, einen weitgehend identischen Gesetzentwurf vor, aber ohne die Zuzahlungsregelung. Doch diese Initiative fand keine Mehrheit im Bundestag. Im April 1996 ergriff dann das BMG erneut die Initiative und führte ein Jahr später eine Anhörung im BMG durch. Nach den Vorstellungen des Ministeriums sollte bei einer neuen Gesetzesvorlage der berufsrechtliche Teil unverändert übernommen werden, allerdings sollten die Psychotherapeuten bzw. Psychotherapeutinnen eigene Kammern bekommen. Verändert wurde der sozialrechtliche Teil: die Psychotherapeuten sollten, anders als im Entwurf der letzten Legislaturperiode vorgesehen, Mitglieder der Kassenärztlichen Vereinigungen werden und über diese mit den Krankenkassen abrechnen. Dies wurde von einem Teil der in der Spiegel AG zusammen arbeitenden Verbände begrüßt, von den anderen aber abgelehnt. Dementsprechend kam es zu einer Teilung der Spiegel AG in die Arbeitsgemeinschaft der Richtlinienverbände (AGR), in der sich die Verbände zusammenschlossen, deren Mitglieder zum Delegationsverfahren zugelassen waren, und die Arbeitsgemeinschaft Psychotherapie (AGPT), die das Delegationsverfahren weiterhin ablehnte und eine Abrechnungsregelung außerhalb der KVen forderte. Beide Gruppierungen waren bei ihren politischen Bemühungen sehr aktiv, aber diese Bemühungen waren aufgrund ihrer unterschiedlichen Zielsetzungen oft widersprüchlich und dadurch weniger effektiv. Die Kernkommission der DGPs und des DPtV entwickelten weiterhin Strategien, um die Gesetzgebung zu fördern. Dazu gehörte unter anderem eine große Demonstration am 14. Mai 1997 in Bonn, organisiert vom BDP.

Am Ende der Legislaturperiode drohte das Gesetz erneut an der Zuzahlungsregelung zu scheitern, an der Minister Seehofer unbedingt festhalten wollte. Der bei einer Besprechung unterbreitete Vorschlag, doch daraus zwei (zusammenhängende) Gesetze zu machen, sodass die SPD diesmal vielleicht zustimmen könne, wurde von ihm aufgegriffen. Am 26.06.1997 stimmte der Bundestag in erster Lesung zu. Nach der zweiten und dritten Lesung im November verwies der Bundesrat das Gesetz an den Vermittlungsausschuss.

Zu diesem Zeitpunkt wurde deutlich, dass aufgrund der Konzentration auf das Sozialrecht keine Forschungsambulanzen vorgesehen waren. Jahrzehnte lang hatte die Forschung Klinischer Psychologen und Psychologinnen darunter gelitten, dass die Forscherinnen und Forscher an Psychologischen Instituten keinen direkten Zugang zu Patienten hatten. Viele wandten sich verhaltensmedizinischen Themen zu, da die Kooperation mit somatischen Kliniken in der Regel leichter war als mit psychiatrischen. Das BMG griff diesen Hinweis insoweit auf, dass sie noch Ausbildungsambulanzen in das Gesetz aufnahm, 
die ebenfalls „vergessen“ worden waren. Wegen der Frage von Forschungsambulanzen sollten wir uns an den Bundesrat wenden. Tatsächlich gelang es, das Gesundheitsministerium Nordrhein-Westfalens von der Notwendigkeit zu überzeugen, so dass im Vermittlungsausschuss noch eine Regelung für Forschungsambulanzen aufgenommen wurde.

Am 12.02.1998 stimmte der Bundestag dem Psychotherapeutengesetz zu, am 6.3.1998 auch der Bundesrat. Genau genommen fehlte eine Stimme für den Einspruch gegen das Zuzahlungsgesetz, sodass endlich, nach 30 Jahren Bemühen, die Psychologischen Psychotherapeuten sowie die Kinder- und Jugendlichenpsychotherapeuten zur selbstständigen und eigenverantwortlichen Ausübung der Heilkunde zugelassen wurden. Nach der Neuwahl des Bundestags beschloss dieser mit seiner neuen Mehrheit von SPD und Grünen, das Zuzahlungsgesetz wieder abzuschaffen, bevor es überhaupt in Kraft getreten war.

Leider waren die Regelungen zur Ausbildung als Bestandteil des Berufsrechts nicht mehr verhandelbar gewesen, sodass das KBV-Modell der privaten Ausbildungsstätten festgeschrieben wurde. Allerdings wurden auch die postgradualen universitären Ausbildungsgänge zugelassen. Diese Regelung entsprach nicht der Ausbildung akademischer Heilberufe, sondern der nicht-akademischer Heilberufe (Heilhilfsberufe) wie Krankenschwestern oder Physiotherapeuten: dreijährige Ausbildung an einer staatlich anerkannten Gesundheits- und Krankenpflegeschule (für die Psychologen: staatlich anerkannten Ausbildungsstätte) und Ablegen einer staatlichen Prüfung.

Die Psychotherapeutinnen und Psychotherapeuten waren in den ersten Jahren nach Inkrafttreten des Gesetzes damit beschäftigt, die berufsrechtlichen Regelungen umzusetzen. Eine Veränderung der Ausbildungsregelung stieß von daher nirgendwo auf Interesse. Erst die Umstellung der universitären Ausbildung auf Bachelor- und Master-Studiengänge regte eine solche Diskussion an (Groeger, 2006).

Das BMG hegte schon aus ordnungspolitischen Gründen selber Zweifel an der Ausbildungsregelung (persönliche Mitteilung) und schrieb ein Forschungsgutachten zur Ausbildung von Psychologischen Psychotherapeuten und Kinder- und Jugendlichenpsychotherapeuten aus, das allerdings im Wesentlichen die inzwischen seit zehn Jahren bestehende Ausbildung befürwortete (Strauß, 2009).

Nur sehr langsam gelang es, Mitstreiter für eine Novellierung der Ausbildungsregelung zu gewinnen. 2009 bildet die Deutsche Gesellschaft für Psychologie eine Kommission „Psychologie und Psychotherapie (PPT)“, die sich mit dem Modell eines sogenannten Direktstudiums der Psychotherapie im Rahmen des Psychologiestudiums mit anschließender Weiterbildung, analog zur Aus- und Wei- terbildung der Ärzteschaft, befasste, entsprechende Vorschläge erarbeitete und verbreitete. Der Widerstand war anfangs groß. Erst 2014 beschloss der Deutsche Psychotherapeutentag, dieses Modell im Grundsatz zu unterstützen. Es dauerte dann noch einmal fünf Jahre, bis 2019 der Bundestag endgültig eine solche Novellierung des Psychotherapeutengesetzes beschloss (s. dazu den Beitrag von Wolfgang Schönpflug in diesem Heft). Damit wurde der Beruf des Psychotherapeuten bzw. Psychotherapeutin endgültig zu einem akademischen Heilberuf, 50 Jahre nach Beginn der Bemühungen.

\section{Literatur}

Daiminger, C. (2007). Eine Erfolgsgeschichte mit Differenzen: Zur Geschichte der Professionalisierung der Verhaltenstherapie und der Deutschen Gesellschaft für Verhaltenstherapie (DGVT) in der Bundesrepublik Deutschland. Tübingen: DGVT.

Deutscher Bundestag. (1973). Enquête über die Lage der Psychiatrie in der Bundesrepublik Deutschland. Zwischenbericht der Sachverständigenkommission zur Erarbeitung der Enquête über die Lage der Psychiatrie in der Bundesrepublik Deutschland (7. Wahlperiode Drucksache 7/1124, 19.10.73).

Deutscher Bundestag. (1975). Bericht über die Lage der Psychiatrie in der Bundesrepublik Deutschland (Drucksache. Nr. 7/4200). Bonn.

Farber, F. R. \& Haarstrick, R. (1989), Kommentar PsychotherapieRichtlinien. München: Elsevier.

Fiedler, P. (2010). Verhaltenstherapie mon amour: Mythos - Fiktion - Wirklichkeit. Stuttgart: Schattauer.

Groeger, W. (2006). Psychotherapie-Ausbildung im Rahmen der Bacherlor-Masterstudienreform. Psychotherapeutenjournal, 4, $340-352$.

Hörmann, G. \& Nestmann, F. (1985). Die Professionalisierung der Klinischen Psychologie und die Entwicklung neuer Berufsfelder in Beratung, Sozialarbeit und Therapie. In M. Ash \& U. Geuter (Hrsg.), Geschichte der deutschen Psychologie im 20. Jahrhundert (S. 252 - 286). Opladen: Westdeutscher Verlag.

Joussen, J. (2004). Berufs- und Arbeitsrecht für Diplom-Psychologen. Göttingen: Hogrefe.

Krampen, G. (1992). Zur Geschichte des Psychologiestudiums. Verfügbar unter: https://www.uni-trier.de/fileadmin/fb1/prof/PSY/ KPW/1992_zur_Geschichte_des_Psychologiestudiums.pdf

Mehring, G. (1992). Mitteilung der Verbände. Vereinigung der Kassenpsychologen. Verhaltenstherapie, 2, 286-287.

Meyer, A.-E, Richter, R., Grawe, K., Graf von Schulenburg, J. M. \& Schulte, B. (1991). Forschungsgutachten zu Fragen eines Psychotherapeutengesetzes. Unveröffentlichtes Gutachten, Universitätskrankenhaus Eppendorf.

Rogers, C. (1951). Client-centered Therapy: Its Current Practice, Implications and Theory. London: Constable.

Rösler, H.-D. (2011). Zur Geschichte der Klinischen Psychologie in der DDR. Report Psychologie (November/Dezember) Verfügbar unter: http://www.report-psychologie.de/heft/archiv/?tx_rpar chive_pi1\%5Barticle\%5D=508\&tx_rparchive_pi1\%5Baction\% $5 \mathrm{D}=$ show\&tx_rparchive_pi1\%5Bcontroller\%5D=Article\&cHash =4bb02251ac283aa2a151ec8a631e1b94

Schildt, H. (2007). Vom „nichtärztlichen“ zum Psychologischen Psychotherapeuten/KJP. Psychotherapeutenjournal, 6 (2), 118 128. 
Schulte, D. (1971), Verhaltenstherapie in der Beratungspraxis. Psychologische Rundschau, 12, $261-275$.

Schulte, D. (1977). So könnte eine Lösung aussehen. Psychologie heute, 4, 66-67.

Schulte, D. (1984). Studium und Berufsvorbereitung. In W. Michaelis \& E. Stephan (Hrsg.), Ausbildungsreform Psychologie (S. 210 - 227). Göttingen: Hogrefe.

Schulte, D. (1987). Modellversuch zur "Weiterbildung in Klinischer Psychologie". In F. E. Weinert \& G. Lüer (Hrsg.), Graduiertenstudien in der Psychologie (S. 122 - 128). Göttingen: Hogrefe.

Schulte, D. (1993a). Modellversuch des Landes Nordrhein-Westfalen an der Ruhr-Universität Bochum. Weiterbildung in Klinischer Psychologie. Schlussbericht. Fakultät für Psychologie der Ruhr-Universität Bochum.

Schulte, D. (1993b). EMNID-Umfrage zur Einstellung der Bevölkerung zur Psychotherapie und zum Psychotherapeutengesetz. Psychologische Rundschau, 44, $201-202$.

Schulte, D. (2019). Der lange Weg zum Psychotherapeutengesetz. Psychotherapie Aktuell, 11 (1), 7-10.

Strauß, B., Barnow, S., Brähler,E., Fegert, J., Fliegel, S., Freyberger, H.J., Goldbeck, L., Leuzinger-Bohleber, M. \& Willutzki, U. (2009). Forschungsgutachten zur Ausbildung von Psychologischen PsychotherapeutInnen und Kinder- und Jugendlichenpsychotherapeut/nnen. Verfügbar unter: https://www.uniklinikum-Jena.de/
mpsy_media/Downloads/Forschung/Endfassung_Forschungs gutachten_Psychotherapieausbildung.pdf

Söhner, F., Fangerau, H. \& Becker, T. (2017). Der Weg zur Psychiatrie-Enquete. Rekonstruktion der politischen Vorbereitung der ersten Enquetekommission des Deutschen Bundestags. Der Nervenarzt, 89, 570 - 578. https://doi.org/10.1007/s00115017-0390-3

Tausch, R. (1968). Gesprächspsychotherapie. Göttingen: Hogrefe Verlag.

Wittchen, H-U. \& Fichter, M.F. (1980). Psychotherapie in der Bundesrepublik. Materialien und Analysen zur psychosozialen und psychotherapeutischen Versorgung. Weinheim: Beltz.

\section{Förderung}

Open Access-Veröffentlichung ermöglicht durch Ruhr-Universität Bochum.

\section{Prof. em. Dr. Dietmar Schulte}

Ruhr-Universität Bochum

Fakultät für Psychologie

Klinische Psychologie und Psychotherapie

44780 Bochum

dietmar.schulte@rub.de 